\title{
EL PROGRAMA DE INFORMÁTICA EDUCATIVA DE LA REFORMA EDUCATIVA CHILENA: ANÁLISIS CRÍTICO
}

\author{
Víctor Cancino Cancino (*), Sebastián Donoso Díaz (**)
}

SÍNTESIS: Se realiza una exposición de la Red Enlaces, que es el programa de informática educativa de la reforma educativa chilena, analizándola desde sus inicios, a comienzos de la década de los 90 , hasta nuestros días. Se describen su concepción inicial y los factores que influyeron en su explosivo crecimiento y expansión, con sus implicaciones en materia de equipamiento, de software y de capacitación, lo que permitirá comprender al lector la experiencia referida en sus aspectos medulares. A partir de ahí se revisan las etapas por las que atraviesa el proyecto, y se identifican sus principales problemas tanto desde el punto de vista de los directivos como de los docentes y los estudiantes, para contrastar estos aspectos con los aportes de los que otras experiencias dan cuenta. Es posible estimar el impacto de la Red en algunas dimensiones: en el plano de la equidad social, ha significado una contribución relevante en la alfabetización inicial de la población más carente de opciones en este campo, incluyendo a padres y a la comunidad; sin embargo, en lo educacional no hay antecedentes generalizables que demuestren las bondades de Enlaces en cuanto a su aportación al currículo y al aprendizaje de los estudiantes. Eso se asocia a debilidades claves, señaladas también por la literatura al respecto, que muestran lo compleja, lenta y costosa que es la correcta apropiación de las TIC, y lo parcial de su incorporación por parte de los docentes en sus prácticas habituales. A tenor de lo expuesto, es fundamental contar lo más pronto posible con estudios que suministren resultados sólidos en los campos indicados, y, al mismo tiempo, rediseñar el proceso de capacitación de los maestros para que la apropiación pueda ser incrementada a una velocidad muy superior a la actual, arbitrando las medidas para que eso ocurra pronto.

(*) Economista, con estudios de postgrado en Política Educacional, profesor de la Facultad de Educación de la Universidad Central.

(**) Doctor en Educación, director del Programa de Magíster en Política y Gestión Educacional del Instituto de Investigación y Desarrollo Educacional de la Universidad de Talca. 


\section{LA REFORMA EDUCATIVA CHILENA Y EL PROYECTO ENLACES}

Con el nombre de «Enlaces» se representa el componente de informática educativa de la reforma educacional chilena. Aunque inicialmente se le menciona como Proyecto Enlaces (1990) y más tarde como Programa Enlaces (1995), e incluso como Red Enlaces, se trata en esencia del mismo elemento, que va adquiriendo una progresión ascendente en la propuesta de la reforma educativa, siendo identificado en el informe sobre Chile de la OCDE (2004) como uno de los «tres componentes estratégicos para cambiar las oportunidades de aprendizaje ofrecidas por el sistema escolar». En concordancia con lo señalado, el «objetivo de Enlaces ha sido la incorporación de las tecnologías de la informática y comunicaciones en el sistema escolar, con el propósito de aportar al mejoramiento de la calidad y equidad» (Hepp, 2003, p. 420).

De la importancia que la Red Enlaces (en adelante Enlaces) tiene en este escenario de reforma caben pocas dudas, al menos en 
cuanto a recursos comprometidos en su desarrollo: «Entre 1990 y 2003 , Chile ha invertido 160 millones de dólares en la introducción de tecnologías de información y comunicación (TIC) en su sistema escolar. Lo logrado se puede resumir en que el $92 \%$ de los estudiantes subvencionados cuenta en su escuela con una sala de computación, el $85 \%$ de los cuales cuenta también con acceso a Internet, y el $76 \%$ de los profesores del país ha recibido capacitación en informática educativa» (OCDE, 2004, p. 24).

Los resultados reseñados se enmarcan en el proceso de reforma educacional que se formaliza como tal en Chile a partir del año 1996, pero que se entiende como el continuador lógico de las políticas educacionales impulsadas desde 1990: "estamos transitando críticamente desde una etapa en la que el énfasis se ponía en los problemas de acceso y cobertura del sistema educativo, a una etapa que enfatiza los problemas de orientación, paradigma y calidad de la educación» (Núñez, 2003, p. 33). El contenido de las políticas educacionales impulsadas en este período (de prerreforma y también de reforma formalizada) «tiene como propósito global responder al problema principal del sistema escolar en la presente década: una baja calidad y la inequidad de la distribución social de sus resultados» (Cox y González, 1997, p. 111). La reforma emplea diversos instrumentos referidos al contexto en el cual se sitúan las políticas, pero la esencia del cambio está en la reforma curricular (Cox, 2003르). La reforma en curso es definida como «un puente entre dos fases revolucionarias, la de la masificación educacional y la de la remodelación inducida por la sociedad del conocimiento, la globalización y las nuevas tecnologías de la información y comunicación» (Núñez; 2003, p. 40). Este puente marca la transición entre diversas etapas de desarrollo de las políticas, correspondiendo a Enlaces un papel significativo en la fase de masificación de la educación, que en algunas dimensiones no está clausurada, como en el ámbito de la brecha digital.

El presente artículo tiene el objetivo de analizar críticamente este componente estratégico de la reforma educativa chilena a tenor de sus fundamentos y de sus etapas de desarrollo, revisando los resultados consignados en las diversas evaluaciones, y, en función de ello, sugiriendo algunas materias sobre las cuales sería importante poner mayor atención. 


\subsection{LA ETAPA INICIAL: ENLACES EN LAS POLITICAS EDUCATIVAS DE COMIENZOS DE LOS AÑOS 90}

Si bien es imprescindible comprender que desde su origen Enlaces estuvo incorporado a un conjunto de estrategias de cambio educativo iniciadas en el año 1990 con la recuperación de la democracia, a saber: P-900, Programa MECE Básica, Estatuto Docente, etc., su rol en la reforma educativa -formalizada el año 1996- va adquiriendo poco a poco mayor significación desde que en 1995 comienza su etapa de masificación (García-Huidobro y Cox, 1999, p. 16).

Con el apoyo del Banco Mundial, el primer gobierno democrático impulsó desde 1991 el programa de Mejoramiento de la Calidad y Equidad de la Educación (MECE), referido al nivel de educación básica, que, a poco de andar, incluyó uno adicional para la enseñanza media. «EI MINEDUC (Ministerio de Educación), a través del Programa MECE, decide comenzar experimentando con el uso de computadores y comunicaciones en escuelas básicas. Esta iniciativa fue enmarcándose gradualmente como un nuevo componente de la reforma bajo el nombre Enlaces» (Hepp, 1999, p. 291).

La idea surgió en un plano experimental, con una iniciativa que se llevó a cabo entre los años 1990 y 1992, a escala reducida de una escuela, mediante un convenio entre el Ministerio y la Pontificia Universidad Católica, cuyo primer resultado fue la constitución de un equipo de trabajo que sentaría las bases del desarrollo posterior de Enlaces. «La idea fue instalar gradualmente una infraestructura que ayudase a unir a las personas y sus proyectos, a intercambiar experiencias educativas entre si, y a reducir el aislamiento de muchas escuelas integrándolas a una red educativa nacional» (Hepp, 1999, p. 291). En esos años de experimentación, con la incorporación de otros centros escolares, se logró dimensionar su potencial pedagógico y comenzó una etapa gradual de incorporación de establecimientos de localidades más alejadas de Santiago. Paralelamente, la dirección técnica de Enlaces, a partir de 1993, se asentó en una universidad de regiones, la Universidad de La Frontera, integrando al equipo que había desarrollado su fase inicial.

En ese período de trabajo ya se había definido el programa de acción. Por una parte, estaba el tema del equipamiento computacional, incluyendo su instalación, puesta en marcha y posterior conexión a Internet; por otra, el tema del software educacional, y, por último, el de capacitación de los docentes. Todo este proceso implicó mayor comple- 
jidad en materia de gestión de Enlaces, como también de sus vínculos con los diversos establecimientos: «En 1995 el MINEDUC, luego de evaluar sus logros a la fecha y ante la creciente demanda de los establecimientos educacionales por contar con tecnología computacional, decidió comenzar una nueva etapa de expansión nacional» (Hepp, 1999, p. 292). De esta forma, Enlaces pasó de 55 establecimientos educacionales en 1994 a 473 en 1996, hasta alcanzar los 3.119 en esta etapa de proyección (año 1998), lo que significó una expansión de 56 veces su tamaño en ese período (Hepp, 1999, p. 293).

En el aspecto técnico implicó generar una red ad hoc, liderada por cinco universidades más el MINEDUC, que, a su vez, se coordinaron con otras tantas universidades para entregar el soporte en materia de apoyo técnico al funcionamiento de la Red, de los equipos computacionales y de la capacitación de los docentes. «El modelo de cambio del proyecto Enlaces fue un modelo de investigación y desarrollo que ha crecido rápidamente, basado en una estructura de apoyo que para el año 2003 comprende 24 universidades, que capacitan profesores y proporcionan apoyo técnico a largo plazo a cada escuela que está en el programa Enlaces» (OCDE, 2004, p. 273).

Esta opción respondió a la creencia en el proyecto, en «los profesores como principales agentes de cambio en el aula, y hacia ellos enfocó su principal estrategia de entrada a las escuelas» (Hepp, 2003, p. 421). Una segunda decisión se refirió a la creación de redes, que más tarde se reforzaron con la posibilidad de Internet; la tercera decisión fue comprender que los computadores eran el medio y no el fin del aprendizaje, razón que la llevó a centrarse en los usuarios más que en la tecnología, lo que se reforzó con la creación de su propio software, proceso complejo que implicó una constante actualización con diferentes decisiones que se adoptaron en la fase siguiente, de las que se da cuenta más adelante.

Las líneas temáticas que fundamentan la incorporación masiva de Enlaces a las escuelas y sobre las cuales se busca impactar con su accionar, son expuestas por Hepp (1999, pp. 296-297) y (2003 pp. 423-425):

- Dimensión calidad: las TIC contribuyen a mejorar los ambientes educativos con recursos de enseñanza-aprendizaje, con artefactos motivadores para los alumnos, con herra- 
mientas comunicacionales y con máquinas para procesar información. Sus principales funciones son:

- Rol pedagógico: profesores y alumnos pueden aprovechar la creciente oferta de recursos electrónicos como material didáctico que apoya los contenidos del currículo.

- Rol cultural, social y profesional: Ias redes fomentan los vínculos personales y el intercambio de experiencias tanto entre profesores como entre alumnos en los ámbitos local, nacional e internacional (Hinostroza y Hepp, 1999).

- Educación para la vida: los jóvenes deben adquirir los conocimientos y las destrezas necesarias en el uso de las TIC, para las nuevas demandas laborales y académicas.

- Gestión: las TIC pueden mejorar la eficacia y la eficiencia de la gestión en todo el sistema educativo:

- Rol de apoyo administrativo: como instrumento para la modernización de los numerosos procesos administrativos de los profesores y de los directivos.

- Equidad: Ias TIC ponen al alcance de las personas una gran variedad y calidad de recursos y de servicios educativos, independientemente del lugar donde éstas se encuentren.

Dos aspectos mencionados en el trabajo de Hepp (1999, pp. 296-297) no son tomados en cuenta en su informe del año 2003, a saber:

- Integración gradual y asistida de la informática en la cultura escolar.

- Integración curricular: integrar la informática educativa desde los objetivos y desde las actividades contempladas en los planes y programas que deben desarrollar los profesores en el aula.

\subsection{LA MASIFICACIÓN DE ENLACES A PARTIR DEL AÑO 1995}

A partir de 1995 Enlaces comenzó su fase de masificación a nivel nacional. Como producto de las fuertes demandas de crecimiento de Enlaces, desde 1996 pasó a encabezar la gestión en desmedro de las 
energías destinadas a explorar nuevas tendencias y al ternativas de uso de las TIC en el aula. En forma paralela eso implicó que, a comienzos de la década del 2000, se traspasaran muchas funciones operativas desde la Universidad de La Frontera a una coordinación de esta materia en el Ministerio de Educación, quedando radicada la función técnica en esa Universidad.

Al mismo tiempo, las acciones de Enlaces se dirigieron a reforzar la red universitaria de asistencia técnica, red que se organizó en torno a seis centros zonales distribuidos a lo largo de todo el país, al que se integraron 18 universidades conformando las 24 instituciones dedicadas a esta tarea, de las cuales dependían cerca de 1.000 profesores capacitadores y más de 150 técnicos para los 8.300 establecimientos escolares del país (Hepp, 2003. p. 428).

Las dimensiones de la tarea de Enlaces se pueden apreciar en la siguiente tabla (núm. 1), (Tomada de Cox, 2003, p. 66).

Tabla 1

Informática Educativa: Cobertura Red Enlaces

\begin{tabular}{|l|c|c|c|c||c|c|c||c}
\hline & $\mathbf{1 9 9 5}$ & $\mathbf{1 9 9 6}$ & $\mathbf{1 9 9 7}$ & $\mathbf{1 9 9 8}$ & $\mathbf{1 9 9 9}$ & $\mathbf{2 0 0 0}$ & $\mathbf{2 0 0 1}$ & $\mathbf{2 0 0 2}$ \\
\hline $\begin{array}{l}\text { Establecimientos } \\
\text { Enlaces }\end{array}$ & 183 & 472 & 1.415 & 3.150 & 4.246 & 5.307 & 6.243 & 7.247 \\
\hline \begin{tabular}{c}
\multicolumn{1}{c}{ Escuelas } \\
Liceos
\end{tabular} & 121 & 311 & 972 & 2.211 & 3.002 & 4.042 & 4.965 & 5.918 \\
\hline \hline $\begin{array}{l}\text { Matrícula en } \\
\text { Enlaces }\end{array}$ & $4 \%$ & $10 \%$ & $28 \%$ & $56 \%$ & $69 \%$ & $80 \%$ & $86 \%$ & $90 \%$ \\
\hline \multicolumn{1}{c|}{ Escuelas } & $3 \%$ & $8 \%$ & $22 \%$ & $49 \%$ & $62 \%$ & $75 \%$ & $84 \%$ & $89 \%$ \\
\cline { 2 - 9 } Liceos & $6 \%$ & $17 \%$ & $43 \%$ & $77 \%$ & $91 \%$ & $91 \%$ & $92 \%$ & $93 \%$ \\
\hline Uso de WWw & & & & & 25.000 & 125.000 & 500.000 & 1.000 .000 \\
\hline
\end{tabular}

Escuelas = enseñanza básica; Liceos = enseñanza secundaria o media.

Los datos muestran el fuerte y sostenido incremento, año a año, en materia de incorporación de establecimientos escolares a Enlaces, un proceso que hasta 1998 significó casi doblar la cobertura del año anterior, comenzando a crecer la tasa a partir de entonces a un ritmo del orden del $20 \%$ anual; al principio la cobertura en enseñanza media fue superior, debido a que el número de establecimientos de este nivel es inferior al de los de enseñanza básica; las consultas a Internet se incrementaron exponencialmente. 
Atendiendo al hecho de que, según Enlaces la clave del cambio educativo está en los docentes, su estrategia de penetración en las escuelas se centró en ellos. Eso implicó diseñar y llevar a cabo un prolongado proceso de capacitación y de acompañamiento a cada establecimiento escolar (Hepp, 1998). Por todo ello, el proceso de capacitación se diseñó para dos años, integrando hasta 20 profesores por cada escuela, actividad que selleva a cabo en sus dependencias; adicionalmente se real iza el proceso de acompañamiento, que se prolonga más allá de la etapa de capacitación.

El programa de capacitación define los logros esperados por un profesor, yse estructura en torno a tres áreas: pedagogía, gestión y cultura informática (Hepp, 2003, pp. 430-431). La primera se ocupa de incorporar las TIC a las prácticas pedagógicas mediante el desarrollo de guías y de modelos para su uso en el aula, y de la reorganización del espacio físico, de los materiales y de las actividades en ese ámbito. La segunda persigue modernizar la gestión docente empleando Internet y software adecuados para preparar clases, guías, presentaciones y material didáctico. Además, se capacita a los docentes para la gestión de bases de datos y para el procesamiento de información básica sobre alumnos, sobre resultados escolares, etc. La tercera, cultura informática, está destinada a instaurar en los docentes las capacidades para mantener y para actualizar los recursos informáticos. Puede decirse que la principal actividad del proyecto, a partir de mediados del año 2003, es el acompañamiento.

J unto a la capacitación docente, Enlaces entrega a cada establecimiento escolar equipos computacionales, software, red de datos, red eléctrica y mobiliario adecuado. Por razones de costo, los equipos para cada establecimiento permiten que los alumnos los utilicen por grupos. Salvo excepciones, no puede trabajar al mismo tiempo todo un curso en un laboratorio computacional; de hecho, la tasa alumnoscomputador es más o menos de 45 estudiantes por equipo (Hepp, 2003, p.235).

Para el software educativo, que es un componente fundamental, la estrategia seguida ha sido capacitar a los profesores para el uso y la selección de los recursos más apropiados. En el 2003 se distribuyeron 58.464 copias de $C D$, con software donado por empresas y por universidades, más el desarrollado por el Instituto de Informática Educativa de la Universidad de La Frontera, a los que se sumaron 60.469 copias de software educativo general, y más de 140.000 licencias de apoyo 
centradas en diversa disciplinas. Además, se desarrolló un portal denominado Educar Chile ( www. educarchile.cl), que tiene diversas secciones de ayuda a los docentes directivos, a los de aula, a investigadores y a los propios estudiantes en distintos aspectos, facilitando el intercambio de guías y de experiencias de trabajo en redes, en Internet, etc. (Hepp, 2003, p. 436).

A partir del 2003 las escuelas fueron abriendo poco a poco los laboratorios a la comunidad, a los padres y a los apoderados, para que se incorporaran al uso de las TIC, en especial al de Internet ${ }^{1}$.

\subsection{LAS DUDAS QUE EL MISMO ENLACES TIENE SOBRE EL PROCESO LLEVADO A CABO}

En primera instancia, es importante anotar que varios de los estudios que reseñan un estado del arte respecto de las variables que tienen mayor impacto sobre los aprendizajes de los escolares chilenos, en especial de aquellos de mayor vulnerabilidad social, dejan establecido el bajo impacto de las TIC, incluyendo el formato Enlaces en el proceso de logros educacionales (Brunner y Elacqua, 2003; Bellei y otros, 2003). Esta situación no es de extrañar, yestá de acuerdo con lo que se esperaba, es decir, que mientras los profesores no cambien sus estrategias docentes, es difícil que se produzcan impactos significativos, como también que otros resultados de estudios internacionales que el mismo Enlaces reproduce en sus documentos, como los de Cuban (2001) y Pelgrum (2001), en los que se da cuenta de lo señalado, indican que: «a) la mayoría de los profesores usa las TIC para mejorar su gestión docente; b) la mayoría de los profesores no transforma sustancialmente al integrar tecnologías en el aula; lo que hace es acomodar la tecnología a su práctica actual. Esta connotación ha hecho replantear los programas de capacitación de profesores, que, junto con introducir las TIC en las escuelas, buscan también modificar las prácticas para mejorar la calidad de los aprendizajes» (Hepp, 2003, p. 432).

En otro texto, el mismo Hepp se explaya sobre el tema «Las TIC han entrado en las escuelas desde hace décadas, pero lo han hecho sólo débilmente al interior del aula, y la constatación (en países desarrolla-

\footnotetext{
${ }^{1}$ Cada escuela dispone durante 10 años de acceso gratuito, y también de tráfico gratuito para operar por Internet.
} 
dos) es que, a pesar de la actual oferta y disposición de tecnología dentro de una escuela, menos del $5 \%$ de los profesores modifica sus prácticas de manera substantiva, como consecuencia de la presencia de las TIC en el aula [... ] Por ciento que los profesores le asignan un gran val or a las TIC como recursos de aprendizaje y como herramientas de gestión y de acceso a nuevas opciones de formación y fuentes de información, pero no hay evidencia de una revolución en las prácticas docentes ni en el sistema educativo en general» (Hepp, 2003ä, p. 444; citando a Vennezky y Davis, 2002).

El análisis proyectivo del trabajo de redes es más auspicioso que lo relacionado con los cambios en las prácticas docentes. El avance tecnológico en materia del ancho de las bandas para el flujo de información, la reducción de costos y el surgimiento de plataformas de trabajo escolar montadas sobre Internet, muestran resultados significativos en materia de uso y de acceso a este recurso, que empujan al sistema escolar de manera eficiente para que se incorpore definitivamente a este proceso (Hepp, 2003, p. 438).

\section{ENLACES: ANÁLISIS DE SUS PRINCIPALES RESULTADOS E IMPACTOS}

En esta sección se analizan los principales resultados alcanzados por el proyecto y algunos de los efectos que han sido registrados en las evaluaciones que se le han practicado. Es importante comprender que, teniendo Enlaces casi una década de operación masiva en el país, y atendiendo a la inversión de recursos que se ha realizado, se requiere disponer de estudios que se refieran al impacto de este proyecto sobre los aprendizajes reales de los estudiantes, fenómeno planteado ya por Arancibia y García (2002), y que no ha sido realizado hasta la fecha mediante mediciones generalizables. Por tanto, se trata de una debilidad cuyas implicaciones son grandes para poder establecer las consecuencias de esta política y de su estrategia.

\subsection{RESULTADOS Y ESTÁNDARES DE COMPARACIÓN}

Como ya fue señalado, las principales áreas de Enlaces son la provisión de tecnología, los contenidos y la capacitación de docentes, ámbitos que por su complejidad se pueden desglosar de la siguiente manera: 
- Tecnología (infraestructura computacional): computadores, impresoras, redes (Intranet e Internet).

- Contenido (software y recursos educativos): portal es educativos: La Plaza y Educar Chile. Recursos digitales: software de productividad estándar, de productividad especializados y software educativo.

- Capacitación: capacitación docente y alfabetización digital a la comunidad.

\subsubsection{Tecnología}

Respecto al área Tecnológica, desde la fase de generalización de Enlaces (1995) se ha mantenido un crecimiento sostenido y significativo, alcanzando «una cobertura de más de ocho mil escuelas y liceos - con 60 mil computadores- sobre un total de 10.476 establecimientos educacionales públicos y subvencionados» (GAD, 2004, p. 19). Ello responde a los siguientes parámetros de distribución de computadores por matrícula del establecimiento:

Tabla 2

Distribución de equipamiento computacional de acuerdo con la matrícula

\begin{tabular}{|l|c|c|c}
\hline Equipamiento & $\begin{array}{c}\text { Menos de } \\
\mathbf{1 0 0} \\
\text { alumnos }\end{array}$ & $\begin{array}{c}\text { Entre } \\
\mathbf{1 0 0} \mathbf{y} \mathbf{3 0 0} \\
\text { alumnos }\end{array}$ & $\begin{array}{c}\text { Más de } \\
\mathbf{3 0 0} \\
\text { alumnos }\end{array}$ \\
\hline Computadores & 3 & 6 & 9 \\
\hline Impresoras & 1 & 2 & 2
\end{tabular}

FUENTE: Estudio de Evaluación de la apropiación y el uso de recursos tecnológicos del proyecto Enlaces por parte de las unidades educativas.

Los datos emanados del Second Information Technology in Education Study (SITES M 1), en los que participó por Chile el Ministerio de Educación, de un total de 28 países, muestran que la disponibilidad de computadores por establecimiento alcanza en promedio 13,1 equipos, de los cuales 7,7 han sido proporcionados por Enlaces ${ }^{2}$. La siguiente

\footnotetext{
${ }^{2}$ Las cifras del estudio muestran que, en enseñanza básica, Chile ocupa el lugar no 20, y en enseñanza media el no 14 entre los 26 países (MINEDUC, 2002, p. 6).
} 
tabla muestra el equipamiento disponible según el tipo de establecimiento:

Tabla 3

Promedio de computadores disponibles

en los establecimientos y porcentaje

\begin{tabular}{|l|c|c|c|}
\hline $\begin{array}{l}\text { Tipo de } \\
\text { Establecimiento }\end{array}$ & $\begin{array}{c}\text { Computadores } \\
\text { aportados por } \\
\text { Enlaces }\end{array}$ & $\begin{array}{c}\text { Total de } \\
\text { computadores por } \\
\text { establecimiento }\end{array}$ & $\begin{array}{c}\text { \% del total } \\
\text { aportado por } \\
\text { Enlaces }\end{array}$ \\
\hline Escuelas & 6,7 & 8,9 & $75 \%$ \\
\hline Liceos & 10,1 & 22,4 & $45 \%$ \\
\hline Todos & 7,7 & 13,1 & $61 \%$ \\
\hline
\end{tabular}

FUENTE: SITES M1 (2002, p. 4).

De los 13,1 computadores por establecimiento, 10,4 están disponibles para el uso de los alumnos. Ello se traduce en enseñanza básica en 50,5 alumnos por cada computador, y en 30,6 en enseñanza media $^{3}$. En términos de calidad, más del $65 \%$ de los equipos tiene procesador Pentium; por ello, «a partir de esta información es posible establecer que Chile cuenta con un alto porcentaje de equipos vigentes. En efecto, en términos generales, Chile supera en porcentaje de equipos con procesadores Pentium a países como Canadá, Israel y Japón o Francia» (MINEDUC, 2002, p. 7).

Respecto al estado del equipamiento, el Estudio SITES (2002, pp. 7-8) establece que un $12 \%$ de los equipos está es desuso, lo que corresponde a un $15 \%$ en enseñanza media, y a un $9 \%$ en básica ${ }^{4}$. Por otra parte, la utilización en promedio de los computadores en los establecimientos alcanza a 28 horas semanales en educación básica, y a 40 en media. De ese tiempo total, el $68 \%$ y el $71 \%$ de básica y media, respectivamente, es utilizado por los alumnos para clases de asignatura

${ }^{3}$ Una definición alternativa de este índice es la razón entre el total de alumnos de cada establecimiento y los computadores disponibles en cada uno de ellos. Eso significa que, para Chile, el promedio subiría a 57,5 alumnos por computador en básica, y a 37,4 en enseñanza media (MINEDUC, 2002, p. 4).

4 Del total de equipos en desuso, el $41 \%$ es por deterioro, el $32 \%$ por obsolescencia, el $14 \%$ por incompatibilidad con la red, el $12 \%$ por otras razones, y un $1 \%$ por equipos que los usuarios no saben cómo utilizar. 
(porcentaje mayoritario), para los talleres extracurriculares, y para uso libre de los estudiantes.

Con relación al acceso y al uso de Internet, Enlaces fue conectando los establecimientos a esta red de manera creciente: «delos ocho mil establecimientos que tienen acceso a equipos computacionales, 4.700 -que agrupan a la mayor parte de la población escolar- tienen acceso a Internet. De estos, a septiembre de 2003, un $80 \%$ poseía conexión conmutada y otro $20 \%$ a banda ancha entre 300-512 Kbps. Esta última cifra revela un importante salto respecto a la situación prevaleciente a principios de 2003 , donde no más del $1 \%$ de los establecimientos conectados a Internet contaba con banda ancha» (GAD, 2004 , p. 19). Así, la cobertura de Internet educativo para establecimientos educacionales en el país alcanza al $60 \%$ de la enseñanza básica y al $68 \%$ de la media. En términos de matrícula, representa el $71 \%$ de la básica y el $76 \%$ de la media (SITES M1, MINEDUC, 2002, p. 31).

Los datos acumulados muestran que Enlaces se ha transformado en un pilar importante de acceso a Internet de niños y jóvenes de menores ingresos, ya que la mayoría de las conexiones a Internet de tipo domiciliario corresponde a familias de los deciles de más ingresos; por lo tanto, como muestra el siguiente gráfico, Enlaces está introduciendo un factor de equidad importante en la reducción de la brecha digital. Tal brecha indica la desigualdad en el acceso a Internet y la calidad de este. Según datos del Informe GAD Chile 2004-2006, «el crecimiento en conectividad ha sido rápido, pero aún existe una importante brecha digital que refleja la desigual distribución de ingresos que persiste en el país. Las estimaciones para 2003 muestran una realidad muy dispar [... ]; en el decil de mayores ingresos el $50 \%$ de los hogares tiene acceso a Internet, lo que contrasta con sólo el 0,7\% para el decil de menores ingresos» (GAD, 2004, p. 10). El mismo Informe da cuenta de que «el país cuenta con una robusta red de telecomunicaciones, que lo coloca en una posición de vanguardia a nivel latinoamericano en cuanto a conectividad, con una penetración de aproximadamente 24 usuarios por cada 100 habitantes, equivalente a 3,7 millones de beneficiarios. En 2003 el acceso a Internet llegó a 500 mil hogares, 100 mil empresas, 4.700 escuelas y liceos, 226 instituciones de educación superior, 1.300 infocentros, todos los servicios del Gobierno central, trece Gobiernos regionales y 320 municipios [...]. Sin embargo, el informe de la Unión Internacional de Telecomunicaciones (UIT) ubica a Chile en el 43. ${ }^{\circ}$ Iugar en materia de acceso digital, lo que representa un significativo rezago respecto de los países desarrollados. Esto ocurre principalmente 
en hogares y empresas, dado que la brecha es menor en cuanto a Gobierno y establecimientos educacionales» (GAD, 2004, p. 13).

\section{Gráfico}

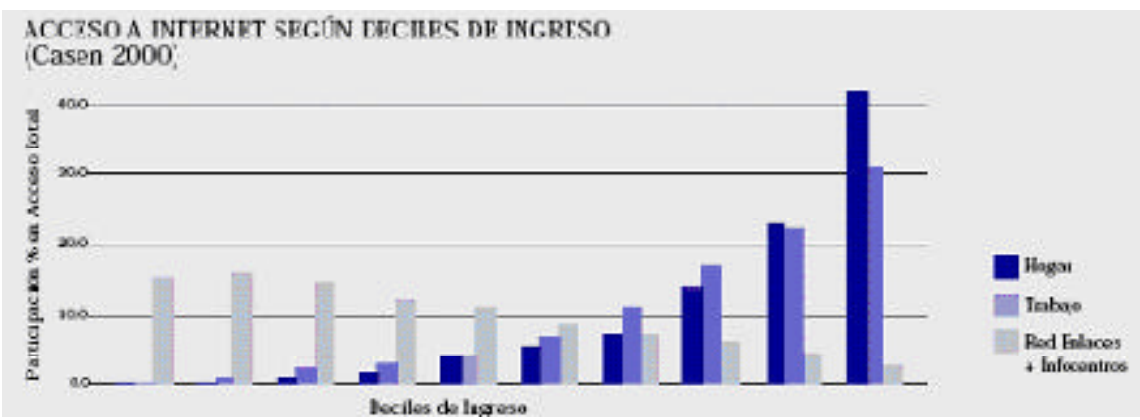

FUENTE: Grupo de Acción Digital (2004, p. 14).

\subsubsection{Contenidos software y recursos educativos}

Un aspecto importante de Enlaces ha sido la entrega de software educativo y de herramientas de productividad: «la provisión de software educativo constituye una dimensión esencial del equipamiento de las escuelas. Cada año se adquiere [... ] nuevo software educativo para cubrir gradualmente todo el espectro curricular» (Hepp (2002, p. 435). En los establecimientos hay diversidad de herramientas disponibles, entre las que destacan el software de productividad estándar, el de productividad especializada y el software educativo. Entre lossoftware de mayor disponibilidad en los establecimientos se encuentran:

Como se señaló, el software educativo de Enlaces tiene por finalidad alcanzar la mayor cobertura posible del currículo actual. La siguiente tabla muestra los porcentajes de alumnos que asisten a establecimientos básicos y medios con software educativo disponible por sectores curriculares:

J unto con distribuir y capacitar a docentes en el uso de nuevas herramientas informáticas, Enlaces desarrolló una plataforma educativa denominada «La Plaza». Más tarde, y debido a la fuerte penetración de Internet, esta plataforma se transformó en el «Portal Enlaces», que, a su vez, derivó rápidamente en un centro de recursos educativos del país. Tal evolución se vio fortalecida por el apoyo que recibió de la mayor empresa de telecomunicaciones de Chile, que donó en 1998 tráfico gratuito de 
Tabla 4

Alumnos de enseñanza básica y media (\%) cuyos encargados de laboratorio declaran tener software para actividades de enseñanzaaprendizaje en al menos un computador (software más utilizado)

\begin{tabular}{|c|l|c|c||c|c}
\hline \multicolumn{2}{|c|}{ Tipo de software } & Básica & $\begin{array}{c}\text { Promedio } \\
\text { Intema- } \\
\text { cional }\end{array}$ & Media & $\begin{array}{c}\text { Promedio } \\
\text { Interna- } \\
\text { cional }\end{array}$ \\
\hline \multirow{2}{*}{$\begin{array}{c}\text { Productividad } \\
\text { Estándar }\end{array}$} & Procesador de texto & 91 & 92 & 88 & 95 \\
\cline { 2 - 6 } & Planilla de cálculo & 85 & 82 & 92 & 94 \\
\cline { 2 - 6 } & $\begin{array}{l}\text { Software de correo } \\
\text { electrónico }\end{array}$ & 47 & 53 & 77 & 64 \\
\cline { 2 - 6 } & $\begin{array}{l}\text { Software de } \\
\text { presentaciones } \\
\text { (power point) }\end{array}$ & 77 & 57 & 87 & 63 \\
\hline $\begin{array}{c}\text { Productividad } \\
\text { Especializados }\end{array}$ & $\begin{array}{l}\text { Programas estadísticos, } \\
\text { matemáticos }\end{array}$ & 16 & 33 & 24 & 37 \\
\hline \multirow{2}{*}{$\begin{array}{c}\text { Software } \\
\text { educativo escolar }\end{array}$} & Juegos educativos & 86 & 51 & 70 & 36 \\
\cline { 2 - 6 } & Enciclopedia en cD-ROM & 86 & 54 & 89 & 52 \\
\hline
\end{tabular}

FUENTE: Elaborada a partir de tabla disponible en Estudio SITES (MINEDUC, 2002, p. 12).

Internet por un período de 10 años, beneficiando a cerca del $70 \%$ de las escuelas. En el año 2001 se creó el «Portal EducarChile», que se desarrolló con base en el Portal Enlaces y con uno de la Fundación Chile, constituyéndose en un centro de recursos educativos que permite el uso del correo electrónico, la participación en foros, el acceso a un número importante de materiales educativos y de investigación pertinentes al currículo nacional, así como acceso a información de actividades de centros de perfeccionamiento y de formación docente.

\subsubsection{Capacitación docente}

Tal como se indicó, incorporar un establecimiento a Enlaces implica que, junto con recibir la infraestructura computacional, el software educativo y el servicio de conexión a Internet, se entrega capacitación y asistencia técnica a través de «las universidades que conforman la red de asistencia técnica de Enlaces y están organizados en torno a un programa central de dos años de duración, seguido de un período de asistencia técnico-pedagógica de largo plazo» (Hepp, 2002, p. 430). Considerando la cobertura de Enlaces, en la actualidad se 
Tabla 5

Alumnos de enseñanza básica y media (\%) cuyos encargados de laboratorio declaran poseer software educativo para ciertos sectores curriculares

\begin{tabular}{|c|c|c|c|c|}
\hline Software & Básica & $\begin{array}{c}\text { Promedio } \\
\text { Interna- } \\
\text { cional }\end{array}$ & Media & $\begin{array}{c}\text { Promedio } \\
\text { Interna- } \\
\text { cional }\end{array}$ \\
\hline Matemática & 64 & 60 & 67 & 52 \\
\hline Física & 9 & 36 & 49 & 45 \\
\hline Química & 6 & 26 & 37 & 30 \\
\hline Biología & 48 & 34 & 76 & 30 \\
\hline Ciencias de la Tierra & 38 & 19 & 40 & 17 \\
\hline Lenguaje y Comunicación & 64 & 45 & 44 & 31 \\
\hline Lengua extranjera & 22 & 40 & 42 & 32 \\
\hline Artes & 40 & 24 & 60 & 18 \\
\hline Historia & 72 & 26 & 80 & 23 \\
\hline Educación Cívica & 4 & 12 & 11 & 10 \\
\hline Economía & 3 & 7 & 9 & 20 \\
\hline Geografía & 55 & 37 & 60 & 25 \\
\hline Áreas vocacionales & 4 & 17 & 34 & 28 \\
\hline Educación en informática & 9 & 42 & 22 & 55 \\
\hline Actividades multidisciplinarias & 6 & 14 & 10 & 14 \\
\hline
\end{tabular}

FUENTE: Disponible en «Enlaces: fortalezas, debilidades y proyecciones futuras» (tomada de estudio SITES).

estima que «hay casi 88 mil profesores capacitados en el uso de TIC, de un total de 120 mil. Todo esto permite afirmar que un $97 \%$ de la población escolar de nuestro país tiene algún tipo de acceso a tecnologías de información y comunicación» (GAD, 2004, p. 19).

En la dimensión de alfabetización digital a la comunidad, Enlaces ha desarrollado la modalidad «abierta a la comunidad», iniciativa del MINEDUC en el marco de una campaña nacional sobre este tema, cuyo objetivo es preparar a la ciudadanía para una sociedad globalizada aprovechando las oportunidades de las TIC, en el sentido de incorporar aquellos sectores marginados a su acceso, en especial a microempresarios, a trabajadores independientes y a madres de familia. Esta campaña 
implica, entre otras estrategias, que los establecimientos escolares abran las puertas de sus salas Enlaces para que, en horarios al ternativos, se capacite a la población señalada en el uso de las TIC. Eso también ha significado mejorar la relación con la comunidad.

Esta iniciativa se inició el año 2002, con más de 500 unidades educacionales del país, lo que permitió la alfabetización digital de más de medio millón de personas. Con vistas a los años 2003 y 2004 , se estima que haya entre 45 mil y 55 mil personas integradas por años ( GAD, 2004, p. 21).

\subsection{DE LOS RESULTADOS A LOS IMPACTOS DE ENLACES: ANÁLISIS DE LOS ESTUDIOS Y DE LAS EVALUACIONES}

La presente sección analiza los principales resultados y los impactos registrado en las evaluaciones de la red Enlaces realizadas entre los años 2000 y 2002, y que corresponden a la fase de apropiación de la Red ${ }^{5}$.

\subsubsection{Esquema de análisis de la etapa de desarrollo de la Red Enlaces}

A efectos de organización analítica de la información recogida, se empleará la secuencia de tres etapas expuesta por Rosas y otros (2002) sobre el proceso de difusión y de implementación de nuevas tecnologías. La primera se identifica como de transferencia, que corresponde a un enfoque radial de transmisión de unidades de información de un organismo central a otros de tipo periférico. En razón de los datos reseñados en la primera parte de este trabajo, se puede afirmar que Enlaces ha alcanzado en buena medida el objetivo de cobertura territorial de la matrícula escolar del país.

Eso ha significado mejorar las oportunidades educacionales, y, por ende, reducir la inequidad en esta materia, al menos en su dimensión escolar, pues a nivel de hogar la situación sigue siendo desequilibrante. El carácter compensatorio de Enlaces forma parte de una política social y educativa de cierta importancia.

\footnotetext{
${ }^{5}$ Se presenta en el Apéndice una breve caracterización de las distintas fuentes de información empleadas.
} 
El tema de acceso a Internet es desde luego más evidente en materia de desigualdad, ante lo cual Enlaces reduce las asimétricas relaciones asociadas al acceso según el nivel socioeconómico de las familias. A este proceso han contribuido otros agentes, públicos y privados, que han permitido que Chile, en el plano escolar, tenga una dotación razonable de equipos en uso, que, en un plano comparativo, lo sitúan entre los primeros cinco países según el estudio SITES M1.

La segunda fase del proceso, la apropiación, corresponde a la valoración que hace la sociedad en términos de aspectos tangibles, con el fin de usar este recurso. A este propósito, los datos muestran que los establecimientos escolares han recibido software educativo tanto general como especializado en tasas superiores a las medias internacionales; sin embargo, la percepción de los usuarios es la de que se trata de un número insuficiente ante la demanda de su uso. Eso podría deberse a problemas relacionados con saber usar tales aspectos, lo que no es un tema menor, pues en la evaluación de directores y de encargados de salas de Enlaces es recurrente que se mencione como insuficiente para alcanzar un desarrollo educativo sustentable de calidad, empleando regularmente dicho recurso para todos los estudiantes, tema que se agrava al referirse al uso de Internet.

Está claro que, más que de un tema técnico desde el punto de vista tecnológico, se refiera a la actitud de los docentes ante el cambio, asunto tratado en la sección 1.3, que confirma que tal etapa se encuentra lograda en parte (podría decirse en una fase inicial), y que si bien se trata de un tema que puede verse facilitado por procesos de capacitación, en el caso chileno, dada la gran proporción de docentes que ha pasado por cursos de capacitación, se trata de algo más complejo, que implicaría cursos impertinentes junto a condiciones inadecuadas de trabajo en los establecimientos, para que los docentes se sientan confiados y motivados para aprender a usarlos, y, en función de ello, incorporarlos a sus prácticas profesionales. Todos estos temas son mencionados por los directivos como de significación, aunque no se precisen criterios para su resolución ${ }^{6}$.

\footnotetext{
${ }^{6}$ Se trata de una manera clásica de enfrentar las dificultades en el sistema educativo chileno. Se menciona la dificultad, pero no se establece un compromiso de solución de los actores con el camino de resolución. Esta visión del problema da cuenta de una desprofesionalización del maestro, que espera que al guien solucioneesa dificultad (el templador o el Ministerio), pero sin su participación directa. Esta concepción también implica que el establecimiento escolar no es una escuela para que aprendan los maestros, sino sólo los estudiantes.
} 
Los datos que reseñan las evaluaciones hacen pensar en la necesidad de revisar en profundidad el proceso de capacitación seguido, y, si persisten estos rasgos, la situación se torna crítica y deberán realizarse cambios significativos en dicha dimensión para acometer el problema bajo estrategias más eficientes.

La tercera etapa corresponde a la de apropiación, que es la que Enlaces impulsa hasta la fecha. Los datos consignados destacan el interés de docentes y de alumnos por emplear lasTIC. Esta predisposición es un punto favorable, aunque se ve rápidamente contrarrestada por las dificultades anotadas para implementar estrategias de trabajo educativo sustentadas en esa alternativa. Si bien los diversos actores señalan una frecuencia de uso importante, los resultados reales dan cuenta de que no hay impactos sustantivos sobre las dimensiones clásicas del aprendizaje.

Uno de los aspectos más difíciles de identificar aisladamente es el impacto de determinadas fisonomías parciales en un proceso complejo y multivariado, como son la dimensión curricular y los aprendizajes de los estudiantes. En algunas de las evaluaciones realizadas se analizan dichos aspectos, sea de manera tangencial o en forma cualitativa; sin embargo, superando las complicaciones metodológicas, que siempre son posibles de resolver bajo determinados parámetros razonables de fiabilidad y de validez, lo cierto es que se deberían haber realizado estudios que permitieran establecer con bases empíricas tales dimensiones, que fueran más allá de las opiniones de los participantes.

\subsubsection{Análisis de los resultados y de los impactos a tenor de las demás informaciones}

Los antecedentes expuestos de manera lata en los informes y en las evaluaciones señaladas a grandes rasgos, son del todo consistentes. Por ejemplo, al medirse el logro de habilidades de los docentes en las áreas de capacitación de Enlaces, el total alcanzado es algo superior al $50 \%$, con predominio de los procesadores de textos sobre las planillas de cálculo, que son los programas con menos logros (estudio de medición de la asistencia técnica).

En otra materia, en la enseñanza media, el porcentaje de alumnos que declara utilizar el computador del establecimiento escolar para trabajar en alguna asignatura no supera el 35\%, y lo normal es que se aproxime al $20 \%$. Se emplea de preferencia para navegar por Internet, 
pero también para uso del procesador de texto con el fin de producir informes.

Analizado el logro de habilidades de los alumnos en diversos software, el que obtuvo mejores estándares $(50 \%)$ fue el procesador de textos, mientras la navegación por Internet fue el más bajo (26\% planilla de cálculo). Esta situación está sesgada desde el punto de vista socioeconómico, ya que el grupo que estudia en la enseñanza privada alcanza estándares del orden del $60 \%$, y obtienen 20 y hasta 30 puntos más sobre los subvencionados privados y los públicos, respectivamente. Eso se asocia sin duda a la situación económica de las familias, y, por tanto, a las diferentes posibilidades de uso que existen de este recurso por parte de aquellas (estudio exploratorio de competencias TIC en alumnos).

Los principales obstáculos identificados por los responsables de los laboratorios Enlaces de enseñanza básica y media se refieren a insuficientes copias de software y a poca adecuación específica, temas que son seguidos por escasos equipos computacionales con acceso a Internet, y por redes locales lentas y poco fiables. Todos estos aspectos confirman que el uso de tales medios presenta cierto grado de problemas importantes, y, atendiendo al hecho de que muchos estudiantes tienen acceso a dichas TIC en su establecimiento escolar, eso hace suponer que el impacto real de Enlaces en materias curriculares y de aprendizaje de los alumnos está muy mediatizado por infinidad de dificultades, y muestra más que nada la importancia de tener mayores antecedentes en una dimensión que es crucial para evaluar la política de informática educativa.

Finalmente, lo señalado se corrobora con la opinión de estudiantes que indican que hay inconvenientes para integrar los computadores en las prácticas pedagógicas en la sala de clases, relacionada también con un pobre logro de las competencias de los docentes en estas dimensiones, forma que los encargados de laboratorios complementan al indicar que existe poca asistencia técnica en materia de operación de los equipos, aspecto que los directores subrayan como prioridad fundamental de asistencia técnica en el uso de las TIC para apoyar el currículo. 


\section{DISCUSIÓN FINAL}

Queda claro que con el programa de informática educativa de la reforma educativa chilena, denominado Red Enlaces, se trató de una iniciativa importante, experimental, que con el correr del tiempo y ante la significativa demanda que tuvo, fue al canzando un desarrollo insospechado, que implicó un crecimiento explosivo de equipamiento, de infraestructura, de capacitación y de apoyo técnico. Este proceso tuvo entonces un fuerte factor emergente, lo que de hecho significó responder -con desfase- a tareas y a expectativas superiores a los recursos disponibles.

A tenor del desarrollo del proyecto y de sus resultados en este campo, es cierto que el tema informático en las escuelas y en el sistema educativo está instalado. También dicha iniciativa ha significado un vínculo importante con la sociedad civil y con las personas, sobre todo de aquellas que no tienen opción de aprender y de usar las Tic en su hogar, siendo un valioso factor de igualación de oportunidades para los alumnos, para sus familias y para la comunidad.

La dimensión apropiación - que es más compleja- está aún por lograrse. Se han producido avances considerables, pero lo cierto es que la integración de las TIC en forma regular y cotidiana al proceso de enseñanza representa todavía una brecha significativa del sistema, salvo excepciones. Sin menospreciar lo alcanzado, lo cierto es que un proceso de inversión del orden de USD 160 millones para una década, que a efectos de mantener actualizada la inversión demandará otros tantos recursos, debería mostrar mayores impactos positivos sobre el cambio en las prácticas docentes y sobre el aprendizaje de los estudiantes.

Desde otra óptica, también es importante detenerse a pensar en sus implicaciones como inversión alternativa de esos recursos, o, mejor dicho, de una parte de ellos en el sistema educacional, pues de no producirse los efectos esperados con la incorporación de las TIC en el sistema educacional y en los aprendizajes logrados, tomarán fuerza aquellas visiones que plantean que el cambio de las prácticas docentes es un tema de complejidad creciente que supera el ámbito de las TIC, siendo posible reasignar tales recursos a dicha tarea.

Eso no implica cuestionar la idea de tener una política de informática educativa, que está fuera de discusión. Se entiende que el debate transita por las diversas opciones que existen en función de las 
prioridades que el país se plantee en materia educacional. En esta área, Chile ha seguido el derrotero de otras experiencias más o menos equivalentes. Los problemas que enfrentamos a escalas de magnitud diferentes son los mismos que los identificados por otros, y pueden resumirse en que las TIC no se han incorporado demasiado a los procesos de enseñanza, que los docentes las emplean como complemento pero que no forman parte del eje estratégico de su docencia, y que si bien el acceso computacional se ha generalizado en la población, su uso más frecuente no es para fines pedagógicos.

En consecuencia, se trata de un proceso de cambio lento y gravoso, tanto por el factor tiempo como por la actualización de recursos de software y de hardware, y cuyo aprovechamiento (rentabilidad) es a largo plazo, costo muy alto para países que deben atender necesidades más urgentes y emplear estrategias más efectivas en el corto y mediano plazo, a fin de incidir de forma positiva en los aprendizajes de sus estudiantes, que hoy son la prioridad.

En un plano educativo estricto, las TIC han mostrado las mismas fortal ezas y debilidades genéricas que la literatura consignaba. Por tanto, los procesos de capacitación diseñados para su apropiación no han permitido reducir las trabas detectadas previamente, lo que insta a revisar estas materias y a proponer correcciones relevantes, pues en caso contrario la inversión realizada corre el riesgo de tener una rentabilidad negativa, sea por uso inadecuado, por desuso o por rápida obsolescencia, producidos por su escasa apropiación.

Un punto que está vinculado con esa discusión se refiere a la capacidad de uso del software para los fines para los que fue diseñado, y, al unísono, su posibilidad de adaptación. Dados los costos de estos procesos, se entiende que corresponde a los docentes, en su calidad de profesionales de la educación, arbitrar las medidas para adecuar y adaptar las TIC y sus elementos a las estrategias de enseñanza empleadas; sin embargo, el constante reclamo de directivos, de responsables y de maestros no muestra sino que esta dimensión debe ser atendida con mucha mayor dedicación, a fin de producir resultados acordes con la inversión. No es factible implementar de manera masiva la incorporación de software específico, pero sí se puede impulsar su adaptación, su innovación e incluso la creación desoftware a nivel local, para atender las diversas particularidades. Eso implica diseñar una política más eficiente en esta materia frente a la vigente en la actualidad. 
Sin llegar a fundamentalismos, el software no es neutro. Por eso, debe tener sinergia con la concepción curricular que se impulsa, y de ahí la importancia de que exista cierta localización para evitar no sólo la dependencia, sino para facilitar un fenómeno fundamental en el uso de cualquier recurso didáctico: su consistencia con los principios curriculares que sustentan la propuesta educativa.

Es cierto que, para poder ahondar en otras dimensiones, se requiere información que hoy -al menos públicamente- no existe. Por eso, la Red Enlaces ha introducido mayor equidad en el sistema educativo en términos de oportunidades educativas, aunque su calidad sea un factor por analizar. Esta contribución es significativa, y los aportes de Enlaces en materia de alfabetización digital de la población más distante de estas opciones son sustantivas. No obstante, su rol responde más al componente social de una política educacional que a su impacto educativo, y no es suficiente desde esta última perspectiva, que es la que fundamenta a la red Enlaces para justificar su continuidad. Se requiere que su propuesta educativa se sincronice con la reforma educativa en los dos planos ejes: su aporte al desarrollo del currículo y su fuerte incidencia sobre los aprendizajes, áreas en las cuales se encuentra en clara deuda, al menos por la ausencia de datos masivos que confirmen su bondad en dichas dimensiones.

\section{APÉNDICE \\ CARACTERIZACIÓN DE LAS DISTINTAS FUENTES DE INFORMACIÓN EMPLEADAS}

Los estudios e investigaciones considerados son seis, que se dirigen a contrastar objetivos diferentes y de naturaleza también distinta, y emplean diversas metodologías y análisis según representatividad y objetivos. En su conjunto entregan datos relevantes, que como tal son permiten formarse una visión bastante completa de las principales dimensiones de la Red. 


\begin{tabular}{|c|c|c|c|c|c|c|}
\hline $\begin{array}{l}8 \\
8 \\
8 \\
5 \\
5 \\
\frac{8}{0} \\
0 \\
\frac{\pi}{8} \\
\frac{8}{8} \\
\frac{8}{8} \\
\frac{8}{2}\end{array}$ & 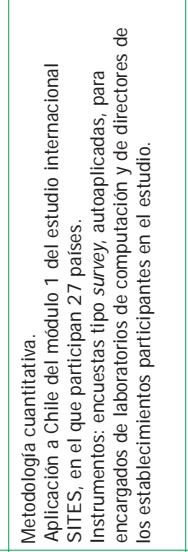 & 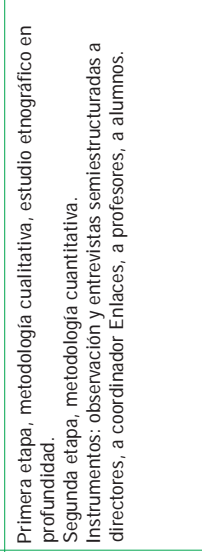 & 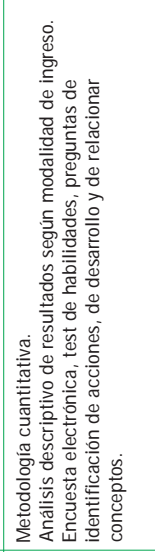 & 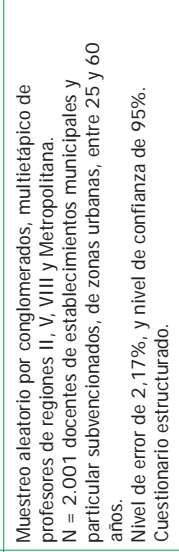 & 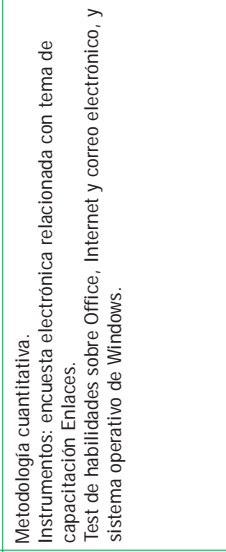 & 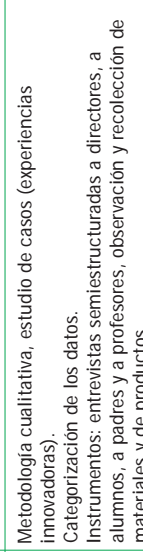 \\
\hline & 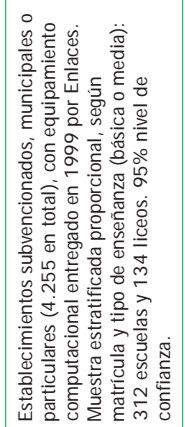 & 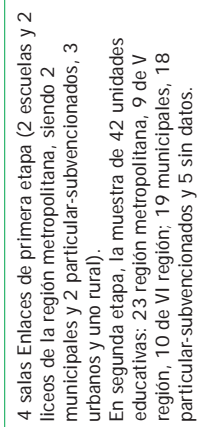 & 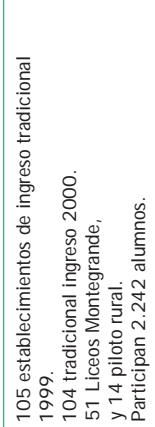 & 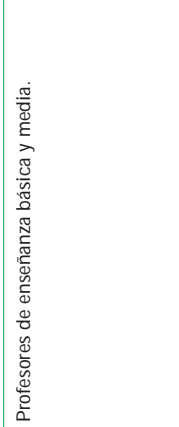 & 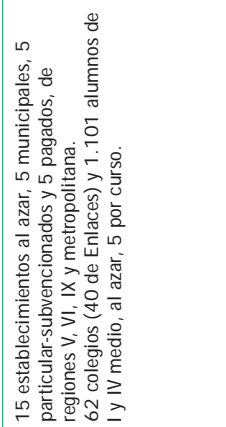 & 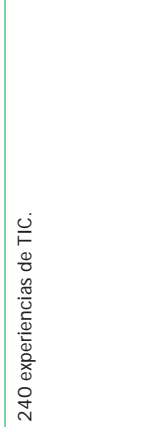 \\
\hline $\begin{array}{l}8 \\
\frac{8}{8} \\
\frac{8}{8}\end{array}$ & 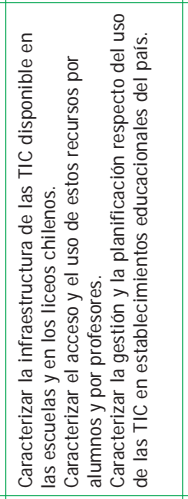 & 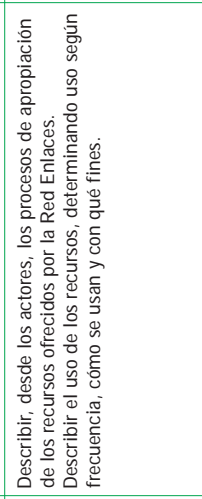 & 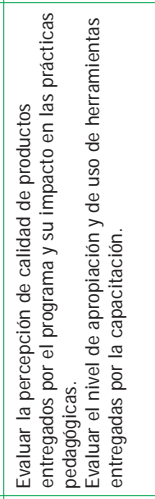 & 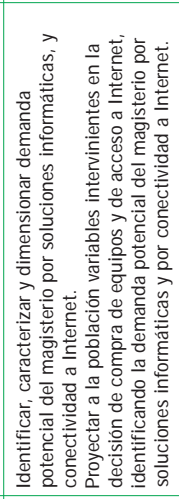 & 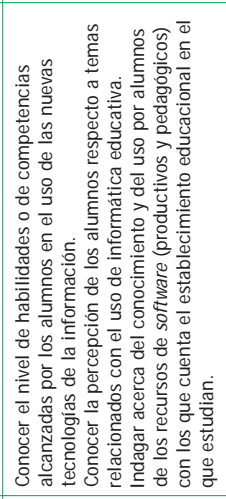 & 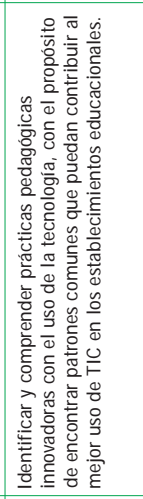 \\
\hline $\begin{array}{l}\delta \\
\frac{\delta}{8} \\
8 \\
8 \\
8 \\
8\end{array}$ & 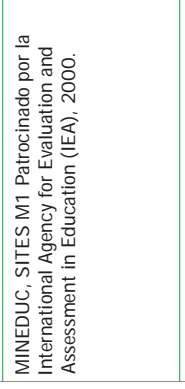 & 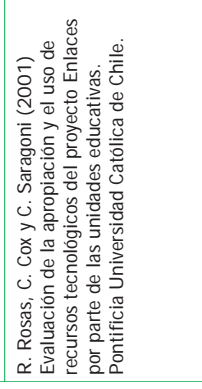 & 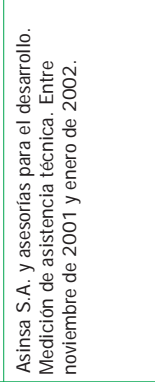 & 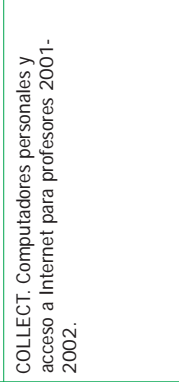 & 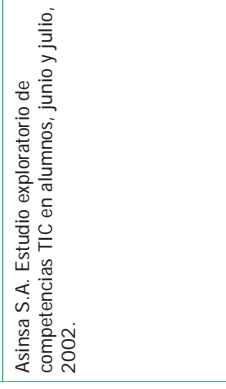 & 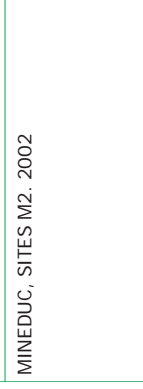 \\
\hline
\end{tabular}




\section{BIBLIOGRAFÍA}

AsinSA S.A. y ASESORÍAS PARA El Desarrollo (2002): Medición de asistencia técnica, Santiago de Chile.

AsINSA S.A. (2002): Estudio exploratorio de competencias TIC en alumnos, Santiago de Chile.

ARANCIBIA, V. yGARCíA, C. (2002): Enlaces: fortalezas, debilidadesy proyecciones futuras, Santiago de Chile.

Bellei, C.; Muñoz, L.; PÉRez, M.; RACZYNSKI, D. (2003): «Escuelas efectivas en sectores de pobreza», en R. Hevia (ed.) (2003): La Educación en Chile Hoy, pp. 347-372, Santiago de Chile, Ediciones Universidad Diego Portales.

BRUNNER, J. J . y ELACQUA, G. (2003): «Factores que inciden en una educación efectiva», en R. Hevia (ed.) (2003): La Educación en Chile Hoy, pp. 45-54, Santiago de Chile, Ediciones Universidad Diego Portales.

COLLECT: (2002) Computadores personales y acceso a Internet para profesores. 2001-2002, Santiago de Chile

Cox, C. (ed.) (2003): Políticas educacionales en el cambio de siglo. La reforma del sistema escolar en Chile, 705 p., Santiago de Chile, Editorial Universitaria.

Cox, C. (2003a): «El nuevo currículo del sistema educacional», en R. Hevia (ed.): La educación en Chile hoy, pp. 117-136, Santiago de Chile, Ediciones Universidad Diego Portales.

COX, C., yGonZáLEZ, P. (1997): «Políticas de mejoramiento de calidad yequidad en la educación escolar en la década de los años 90 », en C. Cox; P. González; I. Núñez, y F. Soto (1997): 160 años de educaciónpública. Historia del Ministerio de Educación,,pp. 101-180, Santiago de Chile.

Cox, C.; GONZÁlez, P.; NúÑEZ, I., y Soto, F. (1997): 160 años de educación pública. Historia del Ministerio de Educación, p. 184, Santiago de Chile.

Cuban, L. (2001): Oversold \& Underused. Computers in the Classrooms, Londres, Harvard University Press.

GARCía-H uidobro, J. E. y Cox, C. (1999): «Capítulo I: La reforma educacional chilena 1990-1998. Visión de conjunto», en J. E. García-Huidobro (ed.): La reforma educacional chilena, pp. 7-46, Madrid, Editorial Popular.

GARCía-H uidobro. J. E. (ed.) (1999): La reforma educacional chilena, 331p., Madrid, Editorial Popular.

GAD (2004). Agenda digital Chile 2004-2006. Grupo de Acción Digital, en www.agendadigital.cl.

HinOSTROZA, J. E. yHePP, P. (1999): «U se of the Web in the Chilean Educational System», J ournal of Computer Assisted Learning, 15, pp. 91-94. 
HEPP, P. (2003): «Capítulo 8. Enlaces: el programa de informática educativa de la reforma educacional chilena», en C. Cox (ed.): Políticas educacionales en el cambio de siglo. La reforma del sistema escolar en Chile, pp. 419-451, Santiago de Chile, Editorial Universitaria.

Hepp, P. (2003a) «Capítulo 6. La educación digital», en R. Hevia, (ed.) (2003): La educación en Chile hoy, pp. 437-448, Santiago de Chile, Ediciones Universidad Diego Portales.

HePp, P. (1999): «Capítulo 14. Enlaces: Todo un mundo para los niños yjóvenes de Chile», en: J. E. García-Huidobro (ed.): La Reforma educacional chilena, pp. 289-303, Madrid, Editorial Popular.

HEPP, P. (1998): «Chilean Experiences in Computer Education Systems», en C. de Moura Castro (ed.): Education in the Information Age, pp. 116-130, Nueva York, BID.

HeVIA, R. (ed.) (2003): La Educación en Chile hoy, 445 p., Santiago de Chile, Ediciones U niversidad Diego Portales.

MINEDUC (2000): Second Information Technology in Education Study SITES M1, Santiago, Ministerio de Educacion de Chile. Patrocinado por la International Agency for Evaluation and Assessment in Education (IEA).

MINEDUC (2002): Estudio Internacional SITES M1: El caso de Chile. Síntesis de resultados y comparación internacional, Santiago de Chile.

NúÑEZ, I. (2003): «Pasado y futuro de la educación chilena», en R. Hevia (ed.): La educación en Chile hoy, pp. 34-44, Santiago de Chile, Ediciones Universidad Diego Portales.

OCDE (2004): Chile. Revisión de las políticas nacionales de educación, 305 p., París, Organización para la Cooperación y el Desarrollo.

Pelgrum, W. (2001): «Obstacles to the Integration of the ICT in Education: Results Form a Worldwide Educational Assessment», Computers \& Education. 37, pp.163178.

ROSAS, R.; COX,C. y SARAGONI, C. (2002): Evaluación de la apropiación y uso de recursos tecnológicos del proyecto Enlaces por parte de las unidades educativas, Pontificia Universidad Católica de Chile.

VENEZKY, R. L. y DAVIS, C. (2003): Lifelong Learning in the Global Knowledge Economy. Challenges for Developing Countries, Washington, Banco Mundial. 